INSULIN SIGNALING

\section{At a snail's pace}

Nat. Struct. Mol. Biol. http://dx.doi.

org/10.1038/nsmb.3292 (2016)

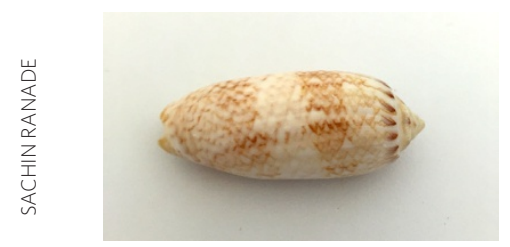

Despite their inoffensive appearance, marine cone snails are fearsome predators that can quickly subdue their prey with complex venoms. Whereas most venom components are neurotoxins that induce pain or paralysis, the venom of some fishhunting snails has been found to contain peptides similar to fish insulins that can induce hypoglycemic shock in prey. One such venomous insulin (Con-Ins G1), produced by Conus geographus, is expressed as a monomer and lacks key residues responsible for hexamerization in vertebrate insulins. The residues missing in Con-Ins G1 are also critical for receptor engagement by vertebrate insulins. Menting et al. noted that this snail insulin is likely to retain the canonical disulfide-bonding pattern of vertebrate insulins, and they found that Con-Ins G1 strongly binds the human insulin receptor and activates signaling. A crystallographic analysis of Con-Ins G1 also revealed its three-dimentional structure and the stabilizing roles of specific post-translational modifications found to potentiate receptor activation. The structure was used to model Con-Ins G1's interaction with the human insulin receptor, which revealed how the small insulin could bind despite its lack of key structural elements present in its human counterpart. This could provide a basis for the future design of ultra-fast-acting insulins.

SL

\section{SYNTHETIC BIOLOGY}

\section{Cholera detectors}

ACS Synth. Biol. http://dx.doi.org/10.1021/ acssynbio.6b00079 (2016)

The presence of Vibrio cholerae-the causative agent of cholera-is currently detected by culture of the bacterium, a slow process, or through the use of molecular methods such as PCR, which can be expensive. To make the detection of $V$. cholerae faster, easier and less expensive, Holowko et al. engineered a strain of Escherichia coli that provides a simple fluorescence-based readout in response to exposure to $V$. cholerae. The presence of the quorum-sensing molecule CAI-1, which is uniquely produced by $V$. cholerae, triggers a dephosphorylation cascade that culminates in repression of transcription from the Qrr4 promoter, which controls expression of an RNA-targeting sequence. When Qrr4 is activated (no CAI-1 present), a CRISPRi system prevents expression of GFP by targeting dCas 9 to the GFP promoter. However, when Qrr4 is repressed, dCas9 targeting is prevented, allowing expression of the GFP reporter. Following optimization of the system guided by in silico modeling, the engineered $E$. coli strain produced an
PROTEOMICS

\section{Fishing for zinc}

The addition of nitric oxide promotes the release of intracellular zinc, which can promote toxicity if unmanaged. Excess zinc is thought to be sequestered in cytoplasmic vesicles; however, a detailed characterization of these vesicles has remained elusive. Miki et al. developed a chemical proteomic platform to identify proteins present under high zinc levels in cells. For this, they used the protein labeling reagent AIZin, which contains a zinc-binding site (dipicolylamine), an acyl imidazole electrophilic reactive group and a fluorophore. AlZin is activated only in the presence of zinc, which allows zinc-responsive protein labeling. The addition of AIZin to glioma cells in the presence of the NO donor resulted in the detection of fluorescent bands that were depleted in the presence of a Zn chelator. Subsequent LC-MS/MS analysis revealed an enrichment of endoplasmic reticulum (ER) and vesicle proteins three hours after NO treatment, suggesting the presence of zinc vesicles. They confirmed that seven candidate proteins including calreticulin and Rab1A exhibited overlapping expression with zinc vesicles in cells. Given the known localization of the identified proteins, the authors propose that the zinc-rich vesicles may be in transit between the ER and Golgi. Although it remains to be determined whether these proteins are involved in the formation of the zinc vesicles, this approach provides a facile tool for the identification of proteins involved in zinc homeostasis on a proteome-wide scale. easily quantifiable fluorescence readout. This system provides a blueprint for the future development of strains that could detect quorum-sensing molecules from other bacteria. Replacing the GFP readout with production of antibacterial compounds could also turn the sensor into a treatment modality.

LONG NON-CODING RNAS

\section{Pulsating RNA motifs \\ Mol. Cell http://dx.doi.org/10.1016/ j.molcel.2016.08.010 (2016)}

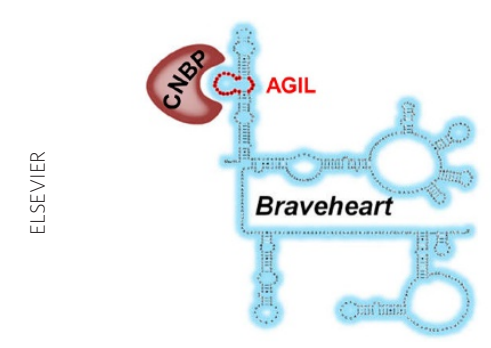

Long non-coding RNAs (lncRNAs) are transcripts of $>200$ nucleotides known to modulate biological processes by targeting gene regulatory pathways. Yet, because of their limited conservation, low abundance and large size, it remains a challenge to determine the molecular mechanisms of lncRNA action in mammalian cells. Xue et al. now define an RNA motif within the mouse Braveheart (Bvht) lncRNA that is required for its function in regulating cardiomyocyte differentiation. Using in vitro chemical probing of full-length and fragment domains of $B h v t$, the authors generated a secondary structure map for the 590-nucleotide Bvht, which features a central five-way junction and an unusual asymmetric G-rich internal loop (AGIL) near the $5^{\prime}$ end of the transcript. Deletion of the AGIL motif within Bvht (dAGIL) by CRISPR/Cas9 genome editing demonstrated that Bvht AGIL is necessary for cardiovascular cell lineage determination. Differential probing of protein microarrays identified proteins - including CNBP, a zinc-finger transcription factor abundant in heart cells - that bind the AGIL motif of Bvht. Knockout studies defined CNBP as a negative regulator of cardiomyocyte development that is antagonistic with dAGIL deletion, supporting a mechanism in which $B v h t$ controls the cardiac cell lineage by selective binding of the negative regulator, CNBP. 